\title{
APPLICATION OF CHEMICALLY ACCELERATED BIOTREATMENT TO REDUCE RISK IN
}

OIL-IMPACTED SOILS

\section{FIRST SEMIANNUAL REPORT}

(SEPTEMBER 1999-MARCH 2000)

\section{Prepared by}

J.R. Paterek

W.W. Bogan

L.M. Lahner

A. May

INSTITUTE OF GAS TECHNOLOGY

1700 South Mount Prospect Road

Des Plaines, Illinois 60018

IGT Project $61121 / 30722$

For

\section{U.S. DEPARTMENT OF ENERGY}

and

\section{GAS RESEARCH INSTITUTE}

DOE Project Manager

Nancy Comstock

GRI Project Manager

John Harju

April 2000 


\section{TABLE OF CONTENTS}

EXECUTIVE SUMMARY $\ldots \ldots \ldots \ldots \ldots \ldots \ldots \ldots \ldots \ldots \ldots \ldots \ldots \ldots$

HIGHLIGHTS $\ldots \ldots \ldots \ldots \ldots \ldots \ldots \ldots \ldots \ldots \ldots \ldots \ldots \ldots \ldots \ldots \ldots$

RESEARCH SUMMARY $\ldots \ldots \ldots \ldots \ldots \ldots \ldots \ldots \ldots \ldots \ldots \ldots \ldots \ldots \ldots \ldots$ iv

RESEARCH ACTIVITIES $\ldots \ldots \ldots \ldots \ldots \ldots \ldots \ldots \ldots \ldots \ldots \ldots \ldots \ldots \ldots \ldots \ldots$

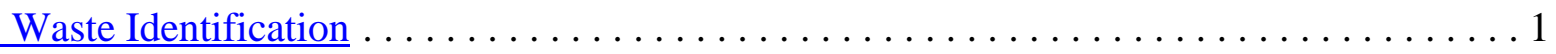

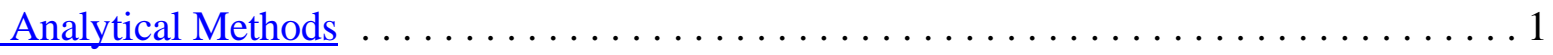

Oil \& Grease Extraction Method for Sludge and Sediment Samples (Method 9071A) . 2

DRO (Diesel Range Organics) Method $\ldots \ldots \ldots \ldots \ldots \ldots \ldots \ldots \ldots \ldots \ldots \ldots \ldots \ldots \ldots \ldots$

GRO (Gasoline Range Organics) \& BTEX (Benzene, Toluene, Ethyl benzene, Xylenes)

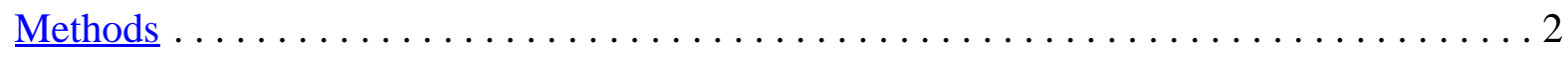

Total Petroleum Hydrocarbons (Method TPH 418.3) $\ldots \ldots \ldots \ldots \ldots \ldots \ldots \ldots$

Polycyclic Aromatic Hydrocarbons (PAH) Method (EPA Methods \#3550A and 8310) . 2

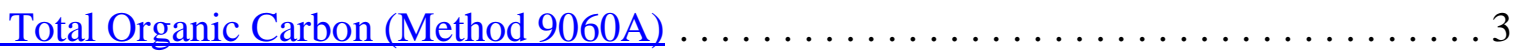




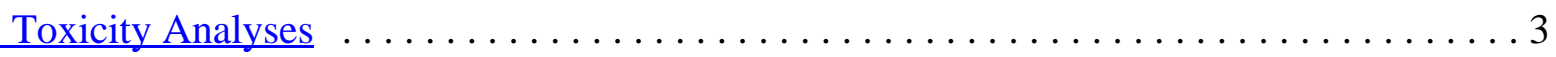

Exploration and Product Soil Collection and Characterization $\ldots \ldots \ldots \ldots \ldots \ldots$.

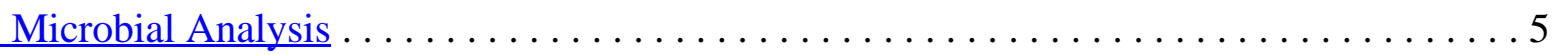

Use of Gaseous Nutrients to Support PAH Degradation $\ldots \ldots \ldots \ldots \ldots \ldots \ldots \ldots \ldots$

Enhancement of PAH Microbial Degradation by Oxidized PAH Intermediates $\ldots \ldots \ldots 8$

Bacterial Survival during Chemical Pretreatment $\ldots \ldots \ldots \ldots \ldots \ldots \ldots \ldots$

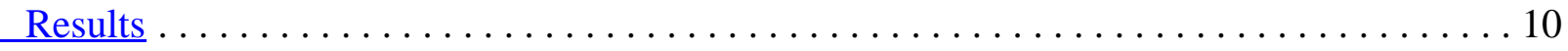

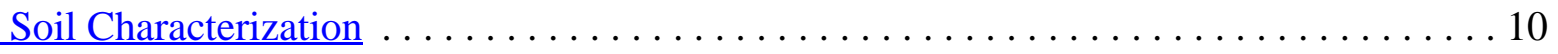

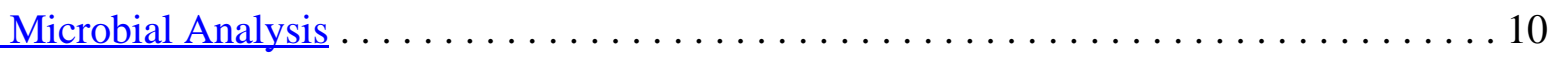

Ability of Gaseous Nutrients to Support PAH Degradation $\ldots \ldots \ldots \ldots \ldots \ldots \ldots \ldots$

Enhancement of PAH Microbial Degradation by Oxidized PAH Intermediates $\ldots \ldots .14$

Bacterial Survival during Chemical Treatment $\ldots \ldots \ldots \ldots \ldots \ldots \ldots \ldots \ldots \ldots$

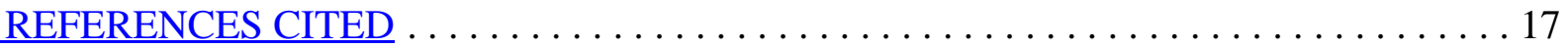




\section{EXECUTIVE SUMMARY}

The results presented within are a summary of research activities from the initiation of a joint project until April 1, 2000 (first semiannual report) between the Institute of Gas Technology (IGT), Department of Energy (DOE), and the Gas Research Institute. Chemically Accelerated Biotreatment (CAB) process that has been proven successful for the treatment of Manufactured Gas Plant (MGP) site wastes therefore the technology is being investigated for application to wastes associated with petroleum/natural gas exploration and production $(\mathrm{E} \& \mathrm{P})$ sites. In addition, the application of $\mathrm{CAB}$ is being evaluation using risk-based analysis along with traditional analytical chemistry testing for endpoint determinations. These results will be applied to the application of environmental acceptable endpoints (EAE) in comparison to strictly analytical assessments. During this first 6 months, work was conducted in five major areas of the project: determination of targeted hydrocarbons; chemical analytical methods initiation and calibration; initiation and calibration of risk (toxicity) methods; cultivation methods for microorganisms degrading elected hydrocarbons; and identification and collection from potential sources of contaminated soil(s).

Based on an initial review of the literature on hydrocarbon compounds and classes in oil and toxicity of various hydrocarbon types (1), we initiated work with a high quality crude oil (Class A); a medium crude oil (Class B); polynuclear aromatic hydrocarbons (PAHs), and volatile hydrocarbons benzene, ethyl benzene, toluene, and xylenes (BTEX). The first tests were carried out with the PAHs and the Class B crude oil.

Methods calibrated for the analyses of the petroleum extracted from the test soils include: Oil and Grease Extraction Method for Sludge and Sediment Samples; DRO (Diesel Range Organics) Method; GRO (Gasoline Range Organics) \& BTEX (Benzene, Toluene, Ethyl benzene, Xylenes) Methods; Total Petroleum Hydrocarbons (Method TPH 418.3); Polycyclic Aromatic Hydrocarbons (PAH) Method (EPA Methods \#3550A and 8310); and Total Organic Carbon (Method 9060A). All extracts and analyses are to be executed by IGT staff using existing instrumentation.

Microorganisms to be used in this project are "naturally occurring" species isolated from contaminated soils, sediments, sludge under study. Methods to isolate microorganisms with the 
capacity to degraded or mineralize PAHs and BTEX were initiated. For PAHs, a novel method presented at the $99^{\text {th }}$ General Meeting of the American Society for Microbiology by J.F. Alley and L.R. Brown (2) has been tested and found effective for the aerobic cultivation of PAHs-degrading microorganisms. Using this method, at least three bacterial isolates capable of growth on fluorene have been purified. One or more of these microorganisms appear to be strains that have not been reported or described. BTEX-degrading microbial cultivations have been initiated using both air and oxidized iron $\left(\mathrm{Fe}^{3+}\right)$ as terminal electron acceptors. Bacterial isolates using both of the acceptors with benzene and naphthalene have been detected.

The project will ultimately focus on the application of CAB with endpoints determined with risk assessment or EAE endpoints, as well as traditional chemical analyses. A number of companies in the USA have been contacted as potential sources for hydrocarbon-contaminated soils. These include: Phillips Petroleum Company http://www.phillips66.com/, Texaco http://www.texaco.com/ and Chevron Petroleum http://www.chevron.com/. Using the website of the Interstate Oil \& Gas Compact Commission (IOGCC) http://www.iogcc.oklaosf.state.ok.us/index.htm sites of production and stripper wells have been identified. Exploration and Production (E\&P) sites located within Illinois have been identified as potential studies sites using Illinois State Division of Oil and Gas http://dnr.state.il.us/mines/dog/welcome.html. Soil from two Illinois sites was collected and testing has been progressing. In addition, a soil from an oil production field in southern California (Long Beach, CA) is under study. 


\section{HIGHLIGHTS}

Research activities in this project for the first two quarters (September, 1999 through April, 2000) generated the following.

C Determination of PAHs, BTEX, and two crude oils as initial contaminants for study.

C Identification and calibration of the chemical analytical methods that will be used for determining baselines for comparison to risk based analyses.

C Water leaching of the Class B crude indicates that toxicity increases are not linear with increase ratios of oil to water.

C Mass transfer of toxicity compounds to water may directly relate to surface area of oil-water interfaces.

C Presence of hydrocarbon-degrading microorganisms is identified in a number of oilcontaminated soils. Isolates maybe unique and un-reported in the literature.

C Gaseous forms of nitrogen and phosphorus stimulate growth and activities of some of the contaminated soils and hydrocarbon-degrading microorganisms.

C Controlled addition of oxidizing agent (Fenton's Reagent) can minimize the damage to microbial communities required for the biodegradation of the hydrocarbons and their intermediates (produced by the oxidizing step).

C Oxidized iron $\left(\mathrm{Fe}^{3+}\right)$ may be an effective terminal electron acceptor for hydrocarbon degrading bacteria. A critical issue as iron is a component of the chemical acceleration technology. 


\title{
RESEARCH SUMMARY
}

Title Application of chemically accelerated biotreatment to reduce risk in oil-impacted soils

\author{
Contractors U.S. Department of Energy \\ DOE Contract No. \\ Institute of Gas Technology \\ GRI Contract No. 8054
}

Principal

Investigator

\section{J. Robert Paterek}

Report Period September 1999 through March 2000

Objective The overall program objective is to develop and evaluate integrated biological/physical/chemical co-treatment strategies for the remediation of wastes associated with the exploration and production of fossil energy. The specific objectives of this project are: chemical accelerated biotreatment (CAB) technology development for enhanced site remediation, application of the risk based analyses to define and support the rationale for environmental acceptable endpoints (EAE) for exploration and production wastes, and evaluate both the technological technologies in conjugation for effective remediation of hydrocarbon contaminated soils from E\&P sites in the USA.

Technical Approach

This project is part of the program of IGT/GRI in an effort to develop and enhance the microbiological approach to the degradation of wastes present at petroleum / natural gas production and exploration sites. It is of primary importance to this program to develop the capability to biologically degrade all the mobile or available fraction of the toxic or hazardous hydrocarbons present at these sites, including the "recalcitrant" aromatic moieties, which includes those compounds containing elements other than carbon in the rings. To achieve this goal, research efforts focuses on enhancing the growth of microbial cultures with capabilities for biodegrading these compounds, aiding microbial activities by sequential or concurrent chemical oxidation of recalcitrant hydrocarbon moieties, determining the mobility and toxicity of the various hydrocarbon components in E\&P wastes, in the conducting parametric studies to maximize detoxification of soils to meet "environmental acceptable endpoints," and providing scientific and engineering supports for future application of in-situ, landfarming, and bioslurry reactor treatment technologies. Research efforts under the GRI project focused on evaluating the performance of selected cultures under a variety of bioremediation conditions in 
simulated "real world" and field conditions found at representative E\&P sites.

Activities Analytical and toxicological techniques and calibrations were initiated this reporting period, as well as the identification and application of microbial cultivation methods for microorganisms that use aromatic hydrocarbons as a carbon/energy source. Preliminary microbial isolates for PAH and benzene- degrading bacteria were executed. Three possible uncharacterized and reported fluorene-degrading microorganisms were isolated. A preliminary water leaching study was carried out and found that toxicity was not linear in relation to increasing oil/water ratios. Evaluation of gaseous nutrients to stimulate the microbial communities was initiated with preliminary indications that these injections of volatile nitrogen/phosphorus compounds may have use in in-situ applications. In one case of pyrene degradation, the gaseous nutrients out preformed the commonly applied soluble forms of nitrogen and phosphate.

Future

Activities Research is planned to continue with the three test soils with biological evaluation being the focus. This should allow us to maximize the effectiveness of both the chemical and biological regimes that comprise the chemically accelerated biotreatment of E\&P wastes. The Mitoscan ${ }^{\mathrm{TM}}$ toxicity analyses system will be incorporated into our analytical schemes and evaluations of contaminated and treated soils. 


\section{RESEARCH ACTIVITIES}

\section{Waste Identification}

The release of hydrocarbon oils into the subsurface causes several problems. Free oils float on groundwater surfaces and can move with groundwater flow. The exploration, production, processing, and transmission of petroleum and natural gas have produced soil, water, and sediments contaminated with a variety of organic and inorganic compounds.

The drilling and operation of gas exploratory wells and the operation of natural gas production wells generates a number of waste materials. They are usually stored and/or processed at the drilling/operations site.

The waste matrices that will be initially examined for the application of IGT's Chemical Accelerated Biotreatment $(\mathrm{CAB})$ process are to be the contaminated soil and sediments from waste drilling and production pits and soil/sediment contaminated by the operation of exploratory drilling or production activities. Nonvolatile components of natural gas hydrates, crude petroleum, and oil-based drilling muds and cuttings are the main source of the organic (hydrocarbons) contaminants in these waste matrixes. The oil-based drilling muds consist of an oil emulsions, organophilic clays, and drilled solids and additives for down-hole rheology and stability, such as fluid loss control materials, thinners, weighting agents, etc.

The contaminants to be monitored and studied for potential remediation using IGT's technologies initially are the total petroleum hydrocarbons $(\mathrm{TPH})$, oil and grease content $(\mathrm{O} \&$ G), BTEX, and polynuclear aromatic hydrocarbons (PAHs). The materials will be monitored in treatment studies of soils, mud, and sediments collected from sites contaminated by exploratory and recovery natural gas wells. This report concentrates on the TPH remedial activities.

Analytical Methods

We have spent some effort during the first three months on method development and validation. In the early period of this study (months 1 through 3 ), we obtaining the necessary 
equipment and reagent for the analyses which are to be conducted during this project. The following is an overview of analytical methods, which will be conducted (either entirely within our laboratory, or in cooperation with the IGT Chemical Research Services department) over the course of this project. This includes methods development and training necessary for our technical team to execute the analyses required throughout this project.

\section{Oil \& Grease Extraction Method for Sludge and Sediment Samples (Method 9071A)}

Necessary solvents, reagents and some equipment (primarily distillation glassware) have been procured. Everything (solvents and equipment) is now available to run this method in-house.

\section{DRO (Diesel Range Organics) Method}

All equipment, solvents, and other reagents needed for this method are available in the lab. Samples will be extracted and analyzed by gas chromatograph (GC).

\section{GRO (Gasoline Range Organics) \& BTEX (Benzene, Toluene, Ethyl benzene, Xylenes)} Methods

The analysis is done using a purge and trap system hooked up to a GC. Because our laboratory is not equipped with a purge and trap system, these samples will be analyzed by IGT's Chemical Research Services.

\section{Total Petroleum Hydrocarbons (Method TPH 418.3)}

Extraction of the samples will be done in our laboratory; analysis and quantification will require the use of a GC-MS, and will, therefore be done by IGT's Chemical Research Services.

\section{Polycyclic Aromatic Hydrocarbons (PAH) Method (EPA Methods \#3550A and 8310)}

Extraction of PAH samples will be done by sonication in our laboratory (a "Sonabox" enclosure has been procured to facilitate this process); samples will then need to be concentrated (a 50-sample Turbovap LV workstation has been purchased, which will greatly streamline and accelerate this process). Analysis will then be done by High Performance Liquid 
Chromatography according to Method \#8310.

\section{Total Organic Carbon (Method 9060A)}

This analysis will be done by IGT's Chemical Research Services.

\section{Toxicity Analyses}

As a preliminary examination into the toxicity of aqueous extracts (simulated leachates) of crude oil, three different extractions were run. Crude oil ( $1 \mathrm{ml}, 10 \mathrm{ml}$, or $25 \mathrm{ml})$ was extracted (via shaking for 24 hours) with $100 \mathrm{ml}$ of distilled water. Each of these extracts was then subjected to Microtox toxicity analysis. The $\mathrm{EC}_{50}$ values thus determined were as follows:

\section{Oil - Water RATiOs}

$1 \mathrm{ml}$ oil extracted $\mathrm{w} / 100 \mathrm{ml} \mathrm{H}_{2} \mathrm{O}$

$10 \mathrm{ml}$ oil extracted w/100 $\mathrm{ml} \mathrm{H}_{2} \mathrm{O}$

$25 \mathrm{ml}$ oil extracted w/100 $\mathrm{ml} \mathrm{H}_{2} \mathrm{O}$
$\mathrm{EC}_{50}\left(5 \mathrm{MIN} . \quad \mathrm{EC}_{50}(15 \mathrm{MIN}\right.$.

EXPOSURE) EXPOSURE)

Thus, it is evident that, even at a oil:water ratio (on a volume basis) of 1:100, a 24-hour aqueous extraction of sweet crude oil resulted in the solubilization of toxic products. However, although extraction of the same oil at the 1:10 or 1:4 ratio yielded an extract which was more toxic (as evidenced by lower EC50 values), this relationship was not linear when compared to the volume of oil extracted. One possible explanation for this observation is that the solubility limit of some subset of the toxic factors is reached at a ratio of oil to water somewhere between 1:100 and 1:10; increasing the amount of oil extracted therefore does not result in further increases in the concentrations of these factors. Alternatively, a greater amount of "clumping" may take place with higher oil:water ratios, which would be expected to impose mass-transfer limitations on the solubilization of the toxic components of the oil.

In the interest of method development and validation, we have run Microtox solidphase tests on soil taken from a New Jersey MGP (manufactured gas plant) site. In order to estimate the accuracy and precision of this method in our hands, we ran this analysis on the 
same soil four times in-house. Our average $\mathrm{EC}_{50}$ value was $1073 \mathrm{mg}$ of soil/L; in other words, slightly over 1 gram of this soil per liter of medium was sufficiently toxic as to reduce the light output from Vibrio fischeri by 50\%. The pertinent data for EC50 values and statistical analyses of our four trials were as follows:

\begin{tabular}{|c|c|c|c|c|}
\hline TriaL & $\mathrm{EC}_{50}(\mathrm{MG} / \mathrm{L})$ & $\begin{array}{c}\text { 95\% CONF. } \\
\text { RANGE }\end{array}$ & $\begin{array}{c}95 \% \text { CONF } \\
\text { FACTOR }\end{array}$ & $\begin{array}{l}\mathbf{R}^{2} \text { OF DATA } \\
\text { POINTS }\end{array}$ \\
\hline$\# 1$ & 1812 & $1136-2893$ & 1.596 & 0.838 \\
\hline \#2 & 806 & $573-1133$ & 1.406 & 0.903 \\
\hline \#3 & 544 & 386-769 & 1.412 & 0.947 \\
\hline \#4 & 1130 & $908-1405$ & 1.244 & 0.958 \\
\hline
\end{tabular}

Sub-samples of the soil were shipped to a contract laboratory $(\mathrm{CH} 2 \mathrm{M}$ Hill, Corvallis, OR) with significant experience in Microtox solid-phase analysis for validation of our results. Their analysis yielded an $\mathrm{EC}_{50}$ value of $1092 \mathrm{mg} / \mathrm{L}$ (95\% confidence interval 821-1454 mg/L), which is clearly in very good agreement with our results. Comparisons of CH2M Hill's 95\% confidence factor (1.332 vs. our average 1.414) and $\mathrm{R}^{2}$ of data points (0.938 vs. our 0.912) with our figures also increases our confidence that we will be able to run this assay over the course of this project and obtain meaningful results.

\section{Exploration and Product Soil Collection and Characterization}

Two representative crude oil-contaminated soils were obtained from the vicinity of wellheads in an oil field in south central Illinois. The first of these, designated Soil AB, was reportedly contaminated with oil pumped from the Salem limestone formation (Mississippian Age), at a depth of approximately 3400 feet. The second soil, designated C, contained oil which originated in the Aux Vases sandstone formation (also Mississippian), at a depth of 2700 feet. Based on conversations with various experts in the oil industry, these petroleum oils should be very representative of crude oils produced throughout the central U.S., including Oklahoma and Texas.

To date, the following baseline physicochemical analyses have been conduced on the 
samples: textural classification, field moisture holding capacity, $\mathrm{pH}$, oil and grease content, and diesel-range organics (DRO). Soil textural classification was done using the hydrometer method (Sheldrick and Wang, 1993). Soil pH was measured according to EPA Method 9045. Oil and grease content of the soils was determined by EPA Method 9071A (four-hour Soxhlet extraction with hexane, followed by evaporation of solvent and gravimetric determination of extracted material). Diesel-range organics measurement was based on the "modified DRO" method published by the Wisconsin DNR, in which samples are dehydrated with sodium sulfate, ground, and Soxhlet extracted (18 hours) in methylene chloride; analysis of the extracts is then conducted by Gas Chromatography.

Analyses for polynuclear aromatic hydrocarbons (PAH) content, total petroleum hydrocarbons (TPH), total organic carbon (TOC), and BTEX are currently underway.

\section{Microbial Analysis}

Isolation of PAH-degrading bacteria from the two soils has been done by standard techniques, based on the formation of "clearing zones" in a layer of pure PAH which has been overlaid onto a plate of agar media previously inoculated with bacteria isolated from the soil in question. This method eliminates the possible introduction of other potential carbon sources (solvents or aerosol carriers) for the bacteria. Briefly, a Pyrex petri dish is filled with sand and heated to a temperature which is sufficiently high for the PAH of interest to sublimate. An aluminum dish containing the pure PAH is placed on the sand. A previously inoculated plate of media is then placed upside down above the dish containing the hydrocarbon, until an even layer of precipitated $\mathrm{PAH}$ forms on the agar. The sublimated plates are incubated at $30^{\circ} \mathrm{C}$ and observed for zones of clearing around the bacterial colonies. A zone of clearing, such as those shown in Figure 1, indicates that the bacterial colony is utilizing the hydrocarbon for its growth; this colony can then be sub-cultured for further study. Colonies with zones of clearing were carefully sub-cultured onto new plates until a pure culture (i.e. one in which microscopic observation shows only one type of bacteria) was obtained.

Bacterial cultures, once isolated, can be tested for degradation of other hydrocarbons. This is being done in 96-well polystyrene plates, in a variation of a previously-published method 
(Gordon, et al. 1993). The wells of the plate contain individual hydrocarbons (anthracene, benzo $[a]$ pyrene, benzo[b]fluoranthene, chrysene, 1,2,5,6-dibenzanthracene, fluoranthene, fluorene, phenanthrene, and pyrene). These hydrocarbons are dissolved in solvent and when transferred to the microplate, the solvent is allowed to evaporate.

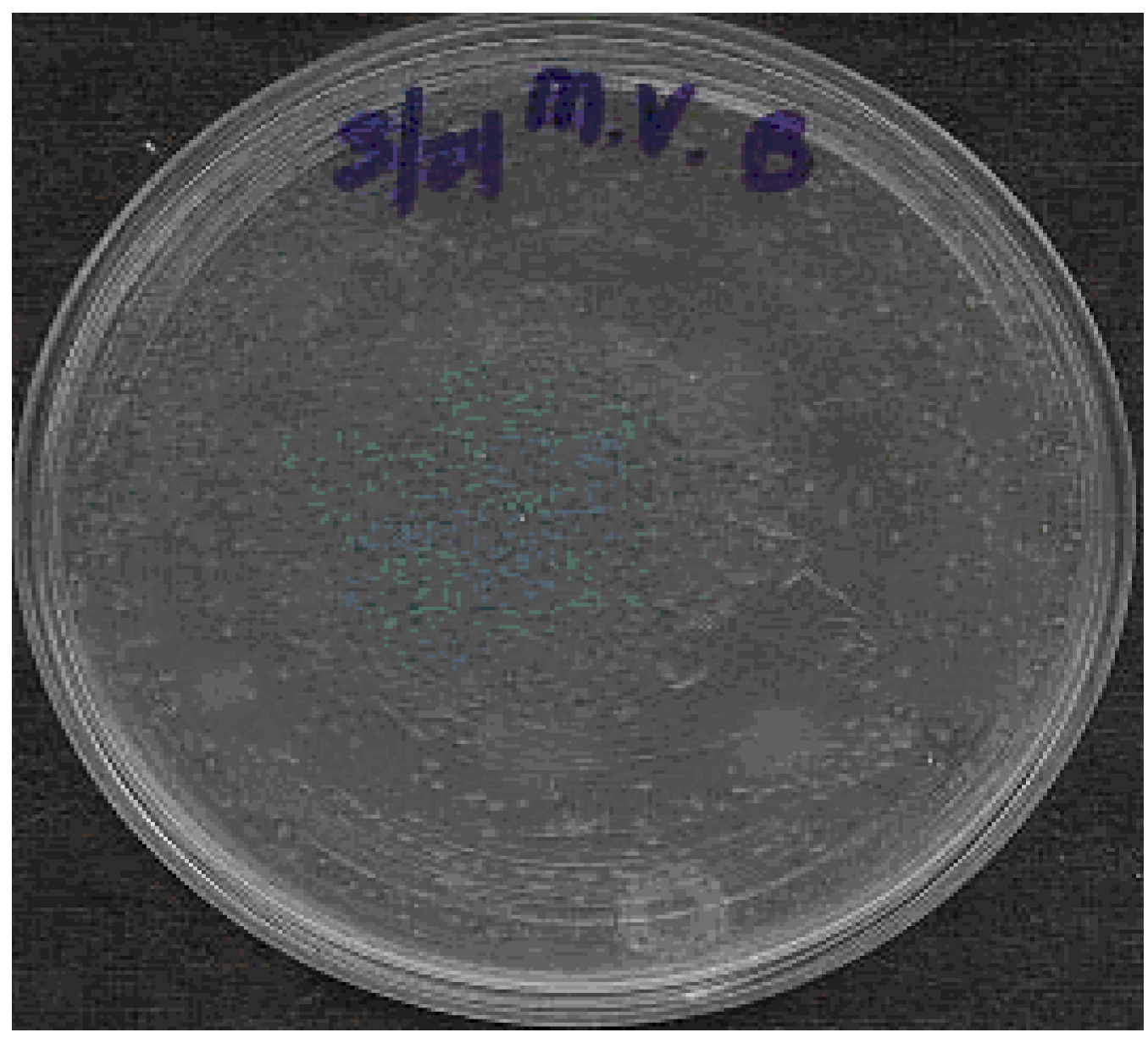

Figure 1. Agar plate containing bacteria from Soil AB, sublimated with phenanthrene. Arrows indicate colonies with zones of clearing, or colonies capable of utilizing phenanthrene as a source of carbon and energy.

Sterile media (which contains no other carbon source for energy and growth of the bacteria) is then added, and the isolated bacteria are inoculated into the wells. Inclusion of the metabolic indicator dye iodonitrotetrazolium (INT) results in a purple color (due to the 
production and precipitation of insoluble formazan) in wells in which cell growth is occurring. The microplates are thus measured for growth by determining absorbance at $405 \mathrm{~nm}$ in an automated microplate reader. This method allows for the simultaneous testing of one or more bacterial isolates on numerous hydrocarbon growth substrates, and under a variety of media conditions - for example, the presence and absence of putative inducers of degradation such as salicylate (see below). Ideally, it should furthermore allow for numerical quantitation of growth on various substrates and direct one-to-one comparisons of growth under different conditions. In practice, we have yet to realize the latter of these, as described below.

Because we are also interested in determining if the isolated bacteria belong to known species, or to a new species, all PAH-degrading isolates are also being subjected to $16 \mathrm{~s}$ ribosomal RNA sequencing (MIDI Labs, Newark, DE). Sequencing results are then compared to both an in-house database (MicroSeq ${ }^{\circledR)}$ maintained by this vendor, as well as the GenBank and Ribosomal Database Project (RDP) database. Species identification is classified as $>99 \%$ match between unknown and published sequences.

\section{$\underline{\text { Use of Gaseous Nutrients to Support PAH Degradation }}$}

As a preliminary experiment to determine whether gaseous nutrients could replace conventional sources of nitrogen (ammonia) and/or phosphorus (inorganic phosphate) under ideal conditions, a liquid-culture study was set up to monitor mineralization of ${ }^{14} \mathrm{C}$-phenanthrene and pyrene under varying nutrient-addition regimes. Soil AB was air-dried and homogenized, and a small amount (500 mg) was used as the inoculum for each independent culture. A concentrated extract of PAHs obtained by extraction of an MGP site soil was also added as a supply of PAH. One set of cultures contained ammonia and inorganic phosphate; in others, ammonia nitrogen was replaced with $\mathrm{N}_{2} \mathrm{O}$ (added to cultures as a gas), and phosphorus was supplied in the form of either tributylphosphate (TBP) or triethylphosphate (TEP). All conditions were run in duplicate. At this stage, both organic phosphates were added in liquid form, and all addition rates of $\mathrm{N}_{2} \mathrm{O}, \mathrm{TEP}$, and TBP were calculated so as to provide the same amount of $\mathrm{N}$ or $\mathrm{P}$ on a molar basis as was included in the ammonia/inorganic phosphate cultures. Mineralization of ${ }^{14} \mathrm{C}$-phenanthrene and pyrene by the bacterial cultures is being 
tracked; based on our prior experience with MGP soils, these experiments will be conducted for a total of approximately four to five weeks. At the conclusion of this experiment, parallel cultures, which received no radiolabeled PAH, will be extracted and analyzed for removal (degradation) of the full range of priority pollutant PAHs. Appropriately killed $\left(\mathrm{HgCl}_{2}\right)$ controls have been included in all studies.

\section{Enhancement of PAH Microbial Degradation by Oxidized PAH Intermediates}

Fenton's reaction, the highly non-specific oxidation of organic compounds by the hydroxyl radical derived from the heterolytic cleavage of hydrogen peroxide by ferrous iron, is being conducted on aqueous solutions of a PAH mixture. The mixed products of this reaction, expected to be a suite of partially oxidized PAH derivatives (phenols, quinones and aromatic acids), will then be added to liquid cultures of bacteria from the oil field soils in order to determine the extent (if any) to which their presence enhances PAH degradation.

\section{$\underline{\text { Bacterial Survival during Chemical Pretreatment }}$}

Two phenanthrene-degrading microorganisms (identification pending) from Soil AB have been isolated. In order to track the survival of these specific organisms during various chemical-oxidation treatments (e.g. $\mathrm{H}_{2} \mathrm{O}_{2}, \mathrm{CaO}_{2}$, peracetic acid), we are attempting to generate antibiotic- (specifically, streptomycin-) resistant mutants of the two species. This is being done according to previously published methods (Davies and Whitbread 1989); briefly, the bacteria of interest are streaked on a plate that contains a gradient of streptomycin (created by layering agar without antibiotic over an antibiotic-containing underlayer of varying thickness. This process is repeated, if necessary, using higher concentrations of streptomycin until a strain is isolated which is capable of growth at an antibiotic concentration which is sufficiently high so as to kill all other organisms present in the soil. Plating of a wash solution of soil inoculated with this strain onto streptomycin-containing plates will therefore result only in growth of the resistant mutant of the PAH-degrading strain. Thus, it will be possible to use standard plate-based methods to enumerate the total heterotrophic bacterial population (by plating soil washes onto standard nutrient agar), total PAH-degrading bacteria (by the sublimation method detailed above), and the total population of the specific strains of interest (by plating on streptomycin- 
containing agar). These counts will be made on one or both of the soils included in this work, both before and after the various chemical treatments listed above. This will provide data on the ability of the species involved in PAH degradation to survive such treatments, both in terms of raw numbers, and relative to the total bacterial population of the soil in question. 


\section{RESULTS}

\section{$\underline{\text { Soil Characterization }}$}

A preliminary textural classification of the two soils has given the following results:

\section{SAMPLE}

Soil AB

Soil C

\author{
$\%$ SAND
}

$72(+/-2)$

$75(+/-2)$
$\%$ CLAY

$7.73(+/-0.01)$

$4(+/-1)$
$\%$ SILT

$20(+/-2)$

$20.81(+/-0.01)$

Thus, each of the two soils would be classified as loamy sands according to the USDA Guide to Textural Classification. Unfortunately, these tests were conducted before the oil and grease analyses; the hydrometer procedure described by Sheldrick and Wang is not recommended for soils with a total organic carbon content of greater than $2 \%$. The TOC of both of these soils will clearly be much higher than this, as the oil and grease content alone (see below) exceeds these values. Thus, we expect that both soils will need to be re-tested following digestion of organic matter. We are currently in the process of initiating this analysis.

The $\mathrm{pH}$ of the soils was found to be as follows: Soil AB was found to have a $\mathrm{pH}$ of 5.9 (+/- 0.2), whereas that of Soil C was found to be $7.6(+/-0.1)$. Thus, Soil AB is somewhat acidic, and Soil $\mathrm{C}$ is very slightly alkaline.

Both soils contained very significant levels of oil and grease, indicative of being significantly impacted by past hydrocarbon spills. Soil AB contained $12.48 \%(+/-0.08 \%, n=3)$ oil and grease by weight. Soil C was actually somewhat higher still, with a total oil \& grease content of $13.7 \%(+/-0.3 \%, n=2)$. Diesel-range organics (DRO) have also been quantitated in the two soils, totaling 960 ppm (+/- 100 ppm, n=2) in Soil AB, and 1540 ppm (+/- 30 ppm, $\mathrm{n}=2$ ) in Soil C.

\section{$\underline{\text { Microbial Analysis }}$}

As Figure 1 shows, we have been able to isolate several PAH-degrading bacteria from 
the E \& P soil samples that are being used in this work. Using plates sublimated with phenanthrene, we have thus far isolated two species, which have been sent out for identification. A minimum of two (perhaps three) further species are in the process of being isolated. Identifications of these species are expected shortly, and will be included in the next progress report. In addition, we have to date isolated and tentatively identified 5 species from a series of PAH- and hydrocarbon-contaminated manufactured gas plant (MGP) sites. Included among these are the following:

ISOLATE

Pseudomonas veronii

Burkholderia sp.

Burkholderia sp.

Sphingomonas sp.

Soil bacterium IS110
LEVEL OF MATCH

Species (MicroSeq \& GenBank)

Genus (MicroSeq \& RDP)

Genus (MicroSeq \& GenBank)

Genus (MicroSeq, GenBank, RDP)

Genus (GenBank)

\section{SOURCE}

New Jersey Hydrocarbon-Contaminated (MGP) soil New Jersey Hydrocarbon-Contaminated (MGP) soil French Hydrocarbon-Contaminated (MGP) soil New Jersey Hydrocarbon-Contaminated (MGP) soil Iowa Hydrocarbon-Contaminated (MGP) soil

All isolates are being evaluated using the microplate method, as described above, to determine their PAH substrate ranges. To date, we have observed growth of the two Burkholderia species, Sphingomonas sp., and Soil Bacterium IS110 on all PAHs tested. In none of these cases were significant growth (as scored by production of purple formazan) detected in wells which received only medium (no carbon source), INT and bacteria. An example of this approach, a microplate inoculated with Pseudomonas sp., is shown in Figure 2. This analysis has been complicated somewhat by the fact that our automated microplate reader has given higher numbers for wells that do not show any purple color than wells that are clearly purple. One possible explanation for this is that the hydrocarbon leaves an opaque film on the bottom of the well, increasing the $\mathrm{A}_{405}$ of that well. Thus, the plates must be visually scored, in order to avoid false positives for growth. New microplates have been purchased that clearly do not react with the solvent used, but these still give a higher readings in the cases of some 


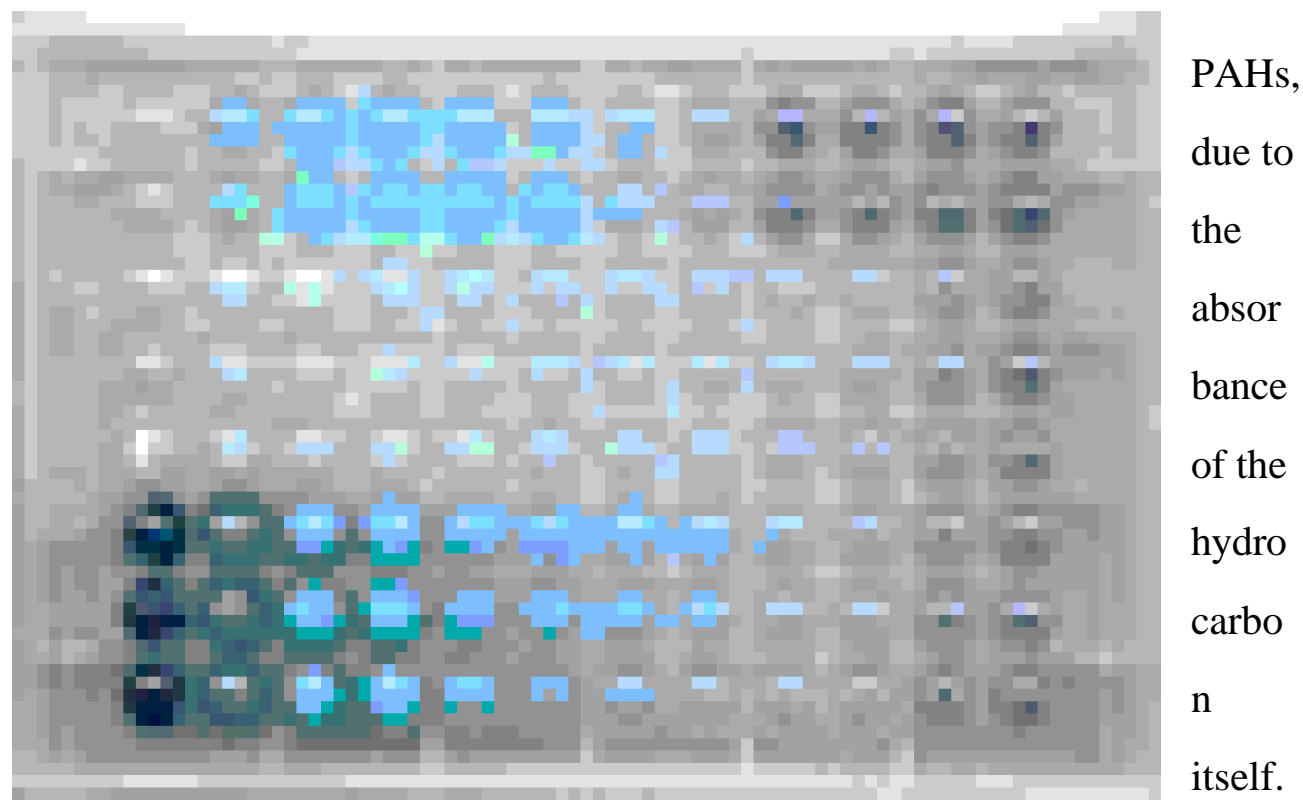

At this point, it is therefore very lt

32

$16 \quad \begin{array}{lll}8 & 4\end{array}$

2

0

Dilution Level

Figure 2.Microtiter Plate Using To Cultivate, Enumerate, and Monitor Activity of Pseudomonas sp. and Other Microorganisms

was difficult in many cases to make quantitative statements comparing growth under different conditions (e.g. on phenanthrene $v s$. benzo[a]pyrene) using this method. 


\section{Ability of Gaseous Nutrients to Support PAH Degradation}

The amounts of phenanthrene and pyrene mineralized by cultures of the microbial community from Soil AB during the first 8 days of incubation are shown below. In the case of phenanthrene, the data thus far indicate that the "traditional" nutrient amendment regime (i.e. solutions of ammonia nitrogen and inorganic phosphate) have given the best results, although several other conditions (most notably $\mathrm{NH}_{4} \mathrm{Cl}$ and triethylphosphate) have also supported significant conversion of phenanthrene to $\mathrm{CO}_{2}$. It seems from this data that either $\mathrm{NH}_{4} \mathrm{Cl}$ or inorganic phosphate can be replaced by a gaseous nutrient; however, it should be noted that replacement of both (i.e. those cultures with $\mathrm{N}_{2} \mathrm{O}$ and either TEP or TBP) results in mineralization totals which are very much reduced from the optimal conditions. In contrast, in the case of pyrene mineralization, it can be seen from the data below that both $\mathrm{NH}_{4} \mathrm{Cl} / \mathrm{TBP}$ and $\mathrm{N}_{2} \mathrm{O} / \mathrm{TBP}$ have (thus far) outperformed the combination of $\mathrm{NH}_{4} \mathrm{Cl}$ and inorganic phosphate. One possible explanation for the significant differences in the patterns of phenanthrene and pyrene mineralization across the spectrum of nutrient conditions studied in this experiment is that different bacterial species are involved in the two processes. Whether or not this is in fact the case, it is noteworthy that, among three soils we have examined in experiments such as these (two MGP site soils and the present E\&P soil), this is the first in which an actual strong "preference" has been shown for an organic phosphate (TBP) by a PAH-degrading soil bacterium.

Clearly, mineralization is not the only acceptable fate for soil pollutants. It will be necessary to determine the actual extent of PAH removal from cultures under the various nutrient regimes before more firm conclusions can be reached regarding the feasibility of using either $\mathrm{N}_{2} \mathrm{O}$ or organic phosphates to support remediation efforts.

\section{Enhancement of PAH Microbial Degradation by Oxidized PAH Intermediates}

Progress in this area has been hampered thus far by the inability to produce sufficient quantities of oxidized PAH via Fenton's reaction. Small-scale aqueous systems (Beltran, et al 1998) have proven unsuitable, due to the extremely low solubility of the PAHs in question. An attempt was made at running Fenton's reaction in a 1:1 mix of water and acetone, based on the 
reported (Bigda 1996) inability of Fenton's reagent to react with acetone; however, this was also unsuccessful. Larger-scale aqueous systems ( $20 \mathrm{~L}$ ) will therefore be used to produce significant quantities of oxidized PAH for these experiments; this is currently underway.

\section{Bacterial Survival during Chemical Treatment}

We are currently actively preparing antibiotic-resistant mutants of PAH-degrading strains from two soils for use in this experiment. This will allow us to isolated these PAHdegrading bacteria selectively in the presence of the microbial population present in the test soils. The first of these is an MGP site, located in Iowa; the second is the southern Illinois oil field site which we are studying as a representative E\&P site. As an illustration of how this is done, preliminary studies on the former of these indicated that concentrations of streptomycin in 
Figure 3. Percent Phenanthrene Mineralized in Mount Vernon Soil

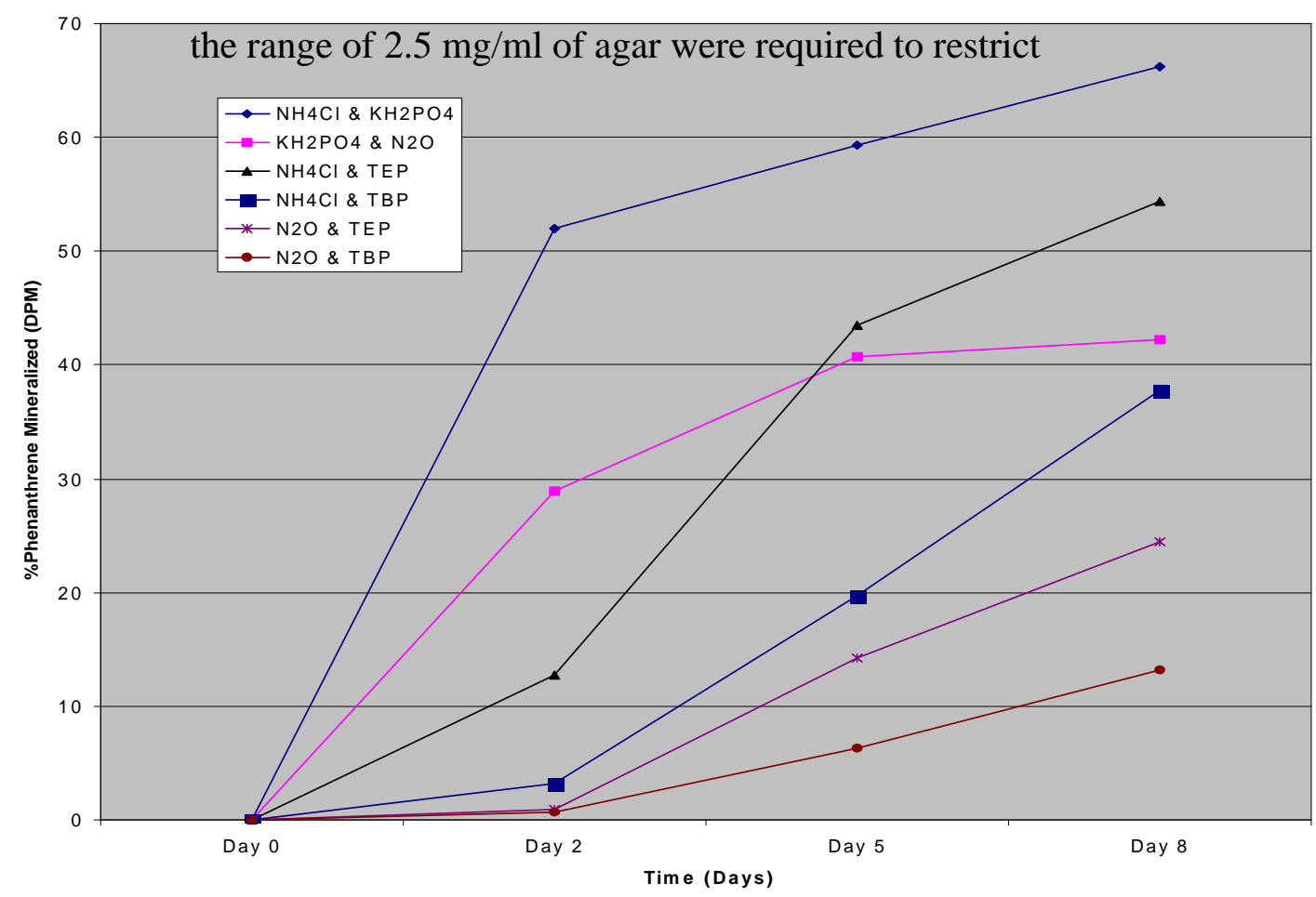

Figure 4. Percent Pyrene Mineralized - Mount Vernon Soil

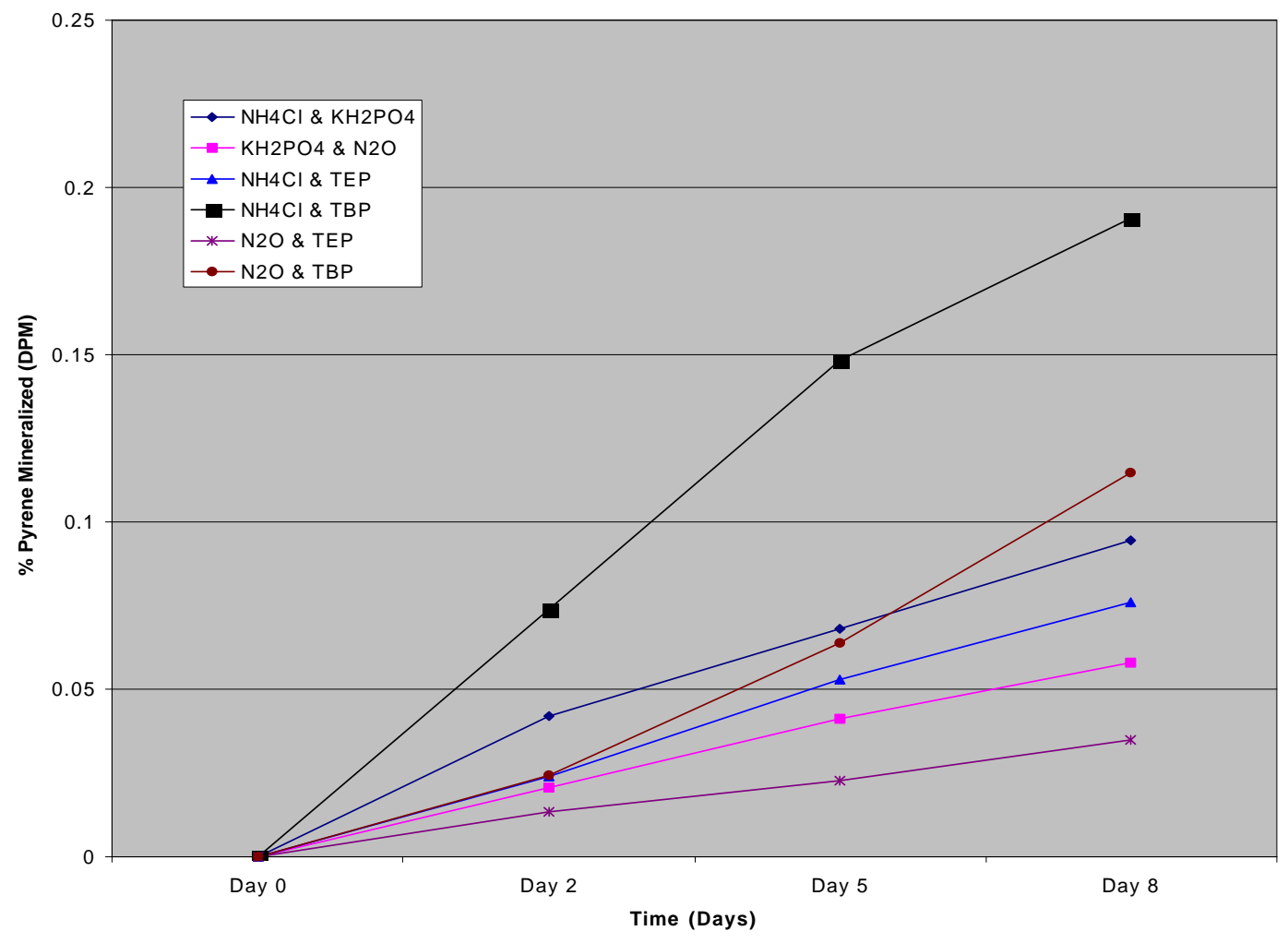


growth of all microorganisms (including wild-type PAH-degraders) in site soil when plated on R2A agar. Thus, we prepared two-layer agar plates by the method of Davies and Whitbread (1989), in which this concentration of streptomycin was present in the lower layer, with a nonstreptomycin-containing overlayer as shown in the following cross-section:

Overlayer - Contains

no streptomycin

Underlayer - Contains $2.5 \mathrm{mg} / \mathrm{ml}$ streptomycin

Figure 5. Detection of Antibiotic Resistant Strains of Soil Microorganisms

After an appropriate equilibration period ( $c a .24$ hours), the surface of the plate contains a gradient of very low (approx. 0) to high (approx. $2.5 \mathrm{mg} / \mathrm{ml}$ ) of streptomycin. PAHdegraders from the site soil are then spread-plated, and (spontaneously arising) mutants are sought which grow nearest the high-antibiotic side of the plate. These can then be tested to ensure that they are capable of growth on the concentration of streptomycin that is required to kill all other microbes from the soil; if necessary, the selection process can be re-run on plates with a higher concentration of antibiotic in the underlayer. Thus far, we are in the process of isolating mutants of one PAH degrader from the Iowa MGP soil, and three from the Illinois E\&P soil. Once isolated, these will be used in experiments such as those described above, and will allow us to assess the survival, during various chemical pretreatment of the soil, specific 
strains of PAH-degrading bacteria, both in absolute terms and compared to that of the microbial community as a whole. 


\section{REFERENCES CITED}

Alley, J.F. and L.R. Brown. 1999. “A novel method for preparing solid microbial media with a virtually water-insoluble carbon and energy source." Preprint and personal communication.

Beltran, F.J., M. Gonzalez, F.J. Rivas, and P. Alvarez. 1998. Fenton Reagent Advanced Oxidation of Polynuclear Aromatic Hydrocarbons in Water. Water, Air, and Soil Pollution 105:685-700.

Bigda, R.J. 1996. Fenton's Chemistry: An Effective Advanced Oxidation Process. Environmental Technology (May/June):34-39.

Davies, K.G. and R. Whitbread. 1989. In Vitro Studies of Siderophore Production by Wild Type and Rifampicin Resistant strains of Fluorescent Pseudomonads. Plant and Soil 116:123125.

Gorden, R.W., T.C. Hazen, and C.B. Fliermans. 1993. Rapid Screening for Bacteria Capable of Degrading Toxic Organic Compounds. Journal of Microbiological Methods 18:339-347.

Lundanes, E. and T. Greibrokk. 1994. "Separation of fuels, heavy fractions, and crude oils into compound classes: A review.” J. High Ressolut. Chromatogr. 17: 197-202.

Sheldrick, B.H. and C. Wang. 1993. Particle Size Distribution. Pages 499-512 in M.R. Carter (ed), Soil Sampling and Methods of Analysis. Lewis Publishers, Boca Raton. 